\title{
Propuesta metodológica para el análisis cartográfico: ejemplo de apreciación de la variable temática «clima» en los Atlas Nacionales de España y Cuba
}

\author{
Ruth Miranda Guerrero y José Sancho Comins*
}

RESUMEN SUMMARY

Con este trabajo pretendemos realizar una aportación a la parte que dentro de la Cartografía Temática se dedica a la expresión cartográfica. A partir de una propuesta metodológica basada en los métodos científico-técnico y perceptivo-

comunicativo, valoramos la variable temática clima de los Atlas Nacionales de España y Cuba con el fin de ofrecer

sugerencias de mejor ejecución. Los Atlas Nacionales, como sabemos, son obras complejas que ofrecen una serie de información que posibilita la caracterización geográfica del territorio al que hacen referencia, en función del perfil temático y la escala de representación. En ellos se presenta una gran variedad de soluciones cartográficas que constituyen por si mismas un interesante objeto de estudio; sin embargo, a pesar de su importancia en distintos ámbitos

(docente, cientifico, cultural,
This work tries to be a methodological contribution to Thematic Cartography in terms of cartographic language. Following a methodology basad on seientific, technique, perceptive and communicative criteria we have analysed the maps that represents the climate variable within the National Atlas of Spain and Cuba. Our objective is to give some suggestions that can be taken into account for improving the cartographic represen ta tion of this variable. National Atlas are very complex documents that offer valuable information in the geographic characterisation of the territory. They usually show a great variety of cartographic solutions that can be considered a very interesting study matter. However, in spite of its importance in differentfields (academic, sefentific, cultural,

\footnotetext{
* Departamento de Geografia, Universidad de Alcalá.
} 
administrativo, etc.), no han sido objeto de estudio per se, de ahi nuestro interés por conocer más de cerca estas obras y plantear una propuesta de análisis que fortalezca el papel de la

Cartografia Temática.

PALABRAS CLAVE
Atlas Nacional de España (ANE),
Nuevo Atlas Nacional de Cuba
(NANC), método cientifico-técnico,
método, perceptivo-comunitacativo,
dimensiones de la comunicación:
Semántica, Sintáctica, Pragmática,
variable temática clima.

institutional, etc), the cartographic language has not been analysed in deep. Hence our interest in studping the above mentioned Atlas and propose a methodology that can help to enrich thamatic cartography.

\section{KEYWORDS}

National Atlas of Spain, New National Atlas of Cuba, scientific-technique criteria, perceptive-communicative criteria, communication dimensions: semantic, syntactic, pragmatic, climate variable

\section{INTRODUCCIÓN}

Los diferentes atlas geográficos que se han elaborado a lo largo del tiempo muestran, de alguna manera, los logros alcanzados en el campo de la Geografía y la Cartografía. Los estudiosos del medio ambiente y cartógrafos de cada época dejaron en ellos su huella impresa. La percepción y conocimiento que se ha tenido del entorno, asi como los avances científicos se manifiestan en los diseños cartográficos y en los contenidos temáticos de estas obras. Las diferentes técnicas de representación e impresión utilizadas han supuesto aportaciones notables a su edición y reproducción, lo que hace que cada atlas o grupo de atlas adquieran una personalidad propia según el momento histórico al que pertenecen.

Raisz, considerado el principal exponente de la Cartografía hasta el final de la década de los cincuenta, decía que un atlas es "una colección de mapas, formando de ordinario un solo tomo, publicado en un mismo idioma, con simbolismos uniformes e idéntica proyección, pero no necesariamente a la misma escala» (Raisz, 1985, pág. 271). Este autor subraya el hecho sustancial: se trata de una colección sistemática de mapas, pero señala que se presentan en un solo idioma. Sabemos que hoy no es así, son muchos los atlas que explican sus contenidos en varios idiomas, como. por ejemplo, el Atlas Nacional de Israel (1985), en él, todos los topónimos están en inglés, y si el espacio lo permitía se han transcrito al hebreo o al árabe, además, los más comunes aparecen en inscripción latina. También Raisz menciona que son obras de un tomo, presentación que actualmente ha sido superada, como ejemplo podemos mencionar los atlas nacionales de Suecia 
(diecisiete volúmenes), de España (cinco volúmenes), de la República Popular China (cinco volúmenes), de México (tres volúmenes), entre otros.

Massó (1993, pág. 97) señala que un Atlas "es ante todo un trabajo de sintesis, un proceso complejo con el objetivo de llegar a conseguir la herramienta de descripción y de localización de los fenómenos fisiográficos y humanos sobre el territorio; herramienta que, por otra parte, nos permite analizar y comprender estos fenómenos". Esta definición enmarca adecuadamente la concepción que actualmente se tiene de este tipo de documentos, quizá faltaria agregar que pueden presentarse en varios soportes, analítico o digital, y en uno o varios idiomas.

En este trabajo nos proponemos analizar este tipo de obras cartográficas desde un doble punto de vista: científico-técnico y comunicativo-perceptivo. La información contenida en los Atlas debe ser precisa, completa, veraz, objetiva, legible y estética. Estas seis características constituyen la base del proceso de comunicación. En él, los sistemas sígnicos, las variables visuales y el diseño global que encierra el conjunto de imágenes de las series cartográficas de cada atlas juegan un papel determinante. Eso es, precisamente, lo que nos ha llevado a realizar este ensayo que pretende marcar pautas de un modelo aplicable a nuevos estudios.

\section{COMENTARIO GENERAL DE LOS DOS ATLAS OWETO DE ESTUDIO}

Hemos elegido para el estudio el Atlas Nacional de España (ANE) y el Nuevo Atlas Nacional de Cuba (NANC). Y, dentro de ellos, el concreto objeto de estudio será una variable temática: el clima. El objetivo general en ambos atlas es similar al que se plantea para todas las obras cartográficas de este tipo: recoger de forma sistemática la información geográfica de la realidad de un país y representarla cartográficamente; sólo así se podrá tener un conocimiento global de sus recursos naturales, de las condiciones sociales, económicas y culturales.

El responsable del proyecto cartográfico del ANE es el Instituto Geográfico Nacional. La estructura temática de éste es de tipo clásico, dividida en XIII secciones, que a su vez se subdividen en 47 grupos temáticos. De acuerdo con dicha estructura, la variable clima pertenece a la sección “ll. El Medio Terrestre». Respecto al diseño del atlas, cabe señalar que cada formato tiene un esquema propio, bien sea como libro encuadernado en 5 volúmenes, cuadernilios o fascículos (51 en total) o sus prototipos multimedia (videodisco y CD-ROM). 
En cuanto al NANC, son encargados del proyecto cartográfico el Instituto de Geografía de la Academia de Ciencias de Cuba y el Instituto Cubano de Geodesia y Cartografía. Su estructura también es clásica, se divide en XXIV secciones temáticas y su diseño corresponde únicamente al de un libro. En este atlas la variable clima se enumera dentro del indica como sección VI.

Respecto al tema elegido, sabemos que es uno de los tradicionales en Cartografía y, por tanto, indispensable en los atlas nacionales. El clima como variable medioambiental de gran relevancia tiene un fuerte impacto en las actividades humanas y su repercusión en el paisaje resulta evidente. En este sentido, la información climática de cada atlas fue de gran interés para poner a prueba nuestra propuesta metodológica, la que exponemos a continuación.

\section{PROPUESTA METODOLÓGICA}

La metodología que proponemos para el análisis comparativo de la variable clima, es una fusión de los planteamientos de Sancho (1997) ${ }^{1}$ y Miranda (1990) ${ }^{2}$. Dicha propuesta cubre dos vertientes, la primera coincide con lo que podriamos denominar método científico-técnico y la segunda con un método comunicativo-perceptivo. El primero consiste en el estudio del contenido y estructura sintáctica, las opciones lingüísticas, el diseño cartográfico y las características técnicas de la obra. Fl segundo método comprende el análisis de los atlas desde el enfoque de las tres dimensiones de la comunicación (Semántica, Sintáctica y Pragmática). Se resumen a continuación las fases de trabajo que suele regirse en cada uno de los métodos ciados y las ideas clave que las soportan.

\section{Método Cientifico-Técnico (Figura 1)}

1. ${ }^{a}$ Etapa: contenido temático. Un proyecto cartográfico tan complejo como un atlas nacional involucra diversas actividades durante su realización, las de carácter geográfico- científico se reflejan en su estructura temática, por ello, esta primera etapa debe dedicarse al estudio de las decisiones de orden semántico.

\footnotetext{
1 El esquema metodológico de SANCHO, C.J. (1997) se ha aplicado como ejercicio docente con alumnos del tercer ciclo del Progiama de Doctorado Cartografía, S.I.G. y Teledetección que el Departamento de Geografía de la Universidad de Alcalá ofrece hace más de 10 años.

MiRANDA, G.R. (1990) hizo la adaptación de la metodologia que el American institute of Graphic Arts (AIGA, 1984) utilizó para la evaluación de simbolos para instalaciones de transporte.
} 


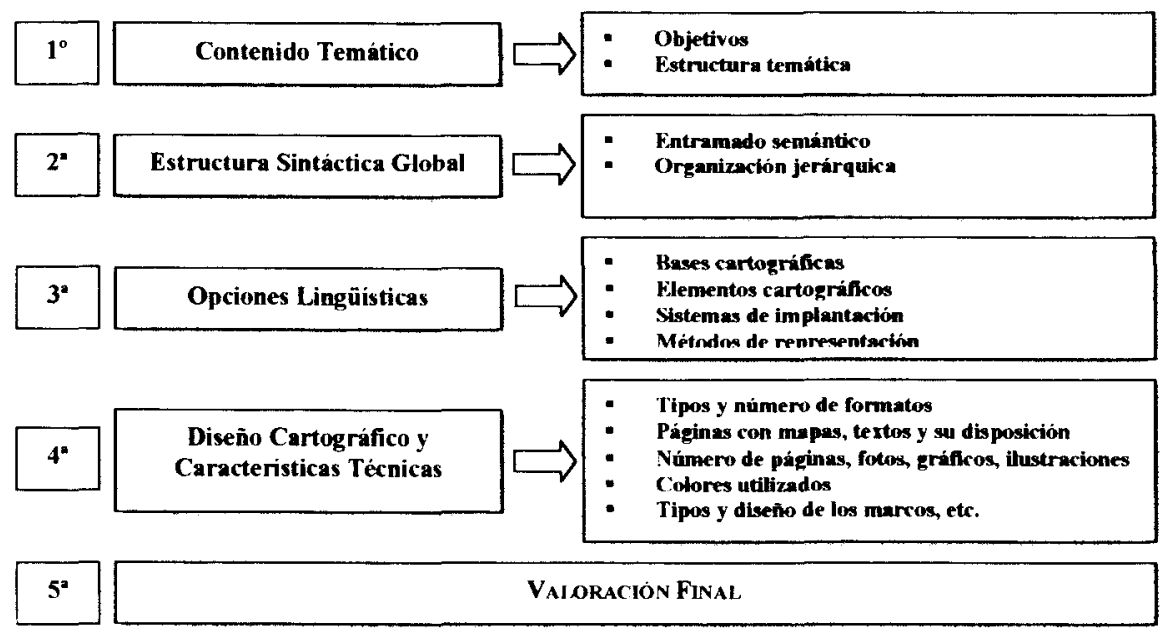

Figura 1. Etapas del método científico-técnico.

2. Etapa: estructura sintáctica general. Se analiza el entramado sintáctico de las obras, su organización jerárquica y las relaciones intercartográficas. Podría también denominarse "análisis de la conformación del discurso", es decir, del tipo de secuencia que concatena los contenidos.

3. Etapa: opciones lingüísticas. Revisamos las bases cartográficas y el empleo del lenguaje cartográfico. Como sabemos, su correcto uso contribuye al cumplimiento de una de las funciones de toda obra cartográfica: comunicar. Un mapa no sólo debe dar prueba de cualidades de fondo, sino, también cualidades de forma, técnica y didáctica, que lo hagan expresivo y legible.

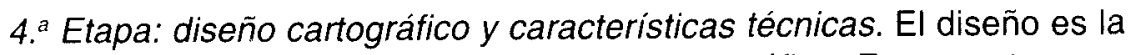
manifestación técnico-artística de una obra cartográfica. En esta etapa se examinan una lista amplia de elementos de orden técnico: cantidad de páginas según sección temática, mapas, textos, fotos, gráficos, ilustraciones; formatos utilizados (marcos, diseño, márgenes, dimensiones); número total de colores; diseño de portadas, cubiertas y sobrecubiertas; ubicación de la información de referencia (páginas, escalas, leyendas, diagramas, textos, tablas, etc.).

5. Etapa: Valoración final. Por último se hace la valoración global cualitativa de las soluciones científicas y técnicas de la obra, esto permitirá ofrecer propuestas de ejecución según se ha creído conveniente. 


\section{Método Comunicativo-Perceptivo (Figura 2)}

Como ya anunciamos, este método se basa en la valoración de los documentos a partir del análisis de las tres dimensiones de la comunicación (Semántica, Sintáctica, Pragmática). Cada dimensión se refiere al carácter que adoptan las relaciones posibles a establecer en un mapa (Figura 2).

La Dimensión Semántica contempla la correlación entre las imágenes cartográficas y su significado. Desde el punto de vista de esta dimensión es importante saber si los datos están bien representados o si los métodos para el tratamiento de la información son los adecuados.

La Dimensión Sintáctica se refiere a las relaciones de los elementos que conforman el mapa. Todos éstos para que adquieran significado lógico deben pertenecer a un sistema sígnico que les permita vincularse y diferenciarse; es decir, con esta dimensión se evalúa la coherencia, armonía y legibilidad de los datos y su representación.

La Dimensión Pragmática atiende las relaciones establecidas entre el mapa y los autores, y entre el mapa y los usuarios. Para la valoración del documento desde esta dimensión consideramos tanto los recursos de la obra - económicos e informativos - como el grupo de usuarios.

Como es bien sabido, la cualidad multiusuario de los atlas es un serio problema al que se enfrentan sus autores dado que no pueden garantizar que las soluciones gráficas dadas cubran todas las necesidades informativas e interpretativas de los diversos lectores. Además, una vez que el

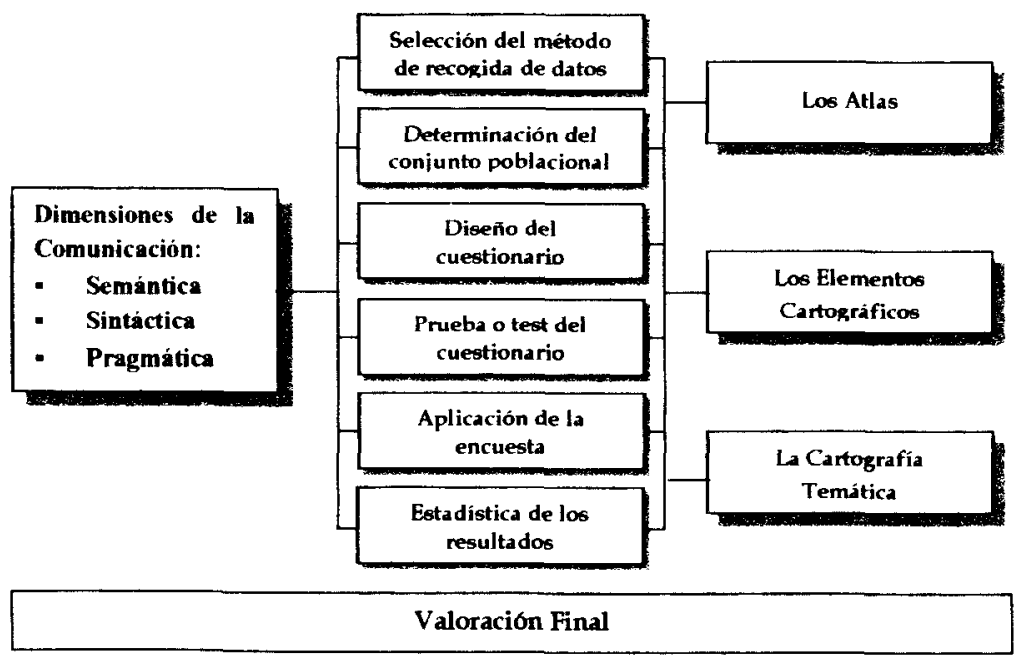

Figura 2. Etapas del método perceptivo-comunicativo. 
producto está en circulación, es raro que los que participaron en su elaboración conozcan cuáles han sido los problemas que los lectores tuvieron al momento de hacer uso del Atlas.

Con este segundo método, en suma, se realiza el análisis de la obra a partir de la opinión de un grupo de usuarios, a los que les pedimos su valoración sobre si las series cartográficas cumplen o no con las tres dimensiones de la comunicación. En nuestro caso, los alumnos de la Universidad de Alcalá constituyeron el conjunto poblacional al que se le aplicó la encuesta.

Aunque se trataba de estudiantes universitarios, es lógico pensar que unos fueron más asiduos usuarios de mapas que otros; por ello, decidimos hacer una revisión de las titulaciones para elegir sólo aquellas que ofrecieran cursos de Cartografía, Geografía o alguna otra Ciencia de la Tierra; seleccionamos finalmente a alumnos de 14 titulaciones distintas. Como el perfil profesional de cada titulación es diferente y, en la mayoría, los cursos de cartografía son de carácter optativo, se nos redujo bastante la posibilidad de que los alumnos tuvieran conocimiento específico sobre temas geográficos o cartográficos. Asi pues, fue necesario dividir la población en dos grupos. En un grupo estarán los alumnos que consultan regularmente obras cartográficas y han seguido enseñanzas de Cartografía, a que catalogamos como «instruidos", y en otro grupo estarían los "profanos» en la materia.

Para homogeneizar aún más los dos grupos generalizamos las titulaciones, fusionamos las especialidades, como por ejemplo, en Magisterio agrupamos Educación Física, Primaria, Infantil, Musical y Lengua Extranjera. De esta forma el universo poblacional sumó 436 alumnos, de los cuáles 324 comprende el grupo de "profanos" y 112 de «instruidos" (Tabla 1).

Tabla 1. Alumnos encuestados agrupados según titulación

\begin{tabular}{|c|l|c|}
\hline \multirow{4}{*}{$\begin{array}{c}\text { Grupo } \\
\text { Profanos }\end{array}$} & \multicolumn{1}{|c|}{ Titulaciones } & Alumnos encuestados \\
\hline \multirow{3}{*}{ (Cuestionario tipo A) } & Turismo & 68 \\
\cline { 2 - 3 } & Magisterio & 132 \\
\cline { 2 - 3 } & Filología & 72 \\
\cline { 2 - 3 } & Historia & 18 \\
\cline { 2 - 3 } & Humanidades & 34 \\
\hline \multirow{3}{*}{$\begin{array}{c}\text { Instruidos } \\
\text { (Cuestionario tipo B) }\end{array}$} & Geografía & $\mathbf{3 2 4}$ \\
\cline { 2 - 3 } & Biología & 9 \\
\cline { 2 - 3 } & Ciencias Ambientales & 15 \\
\cline { 2 - 3 } & Cartogratía y Geodesia Subtotal & 75 \\
\hline \multicolumn{2}{|c|}{ Total } & $\mathbf{1 1 2}$ \\
\hline \multirow{2}{*}{$\begin{array}{l}\text { Nota: Los totales que se presentan son la suma únicamente de los alumnos presentes el } \\
\text { dia de la encuesta no del total de matriculados en cada titulación }\end{array}$} \\
\hline
\end{tabular}


Al tener dos grupos diferentes, también fue necesario diseñar dos tipos de cuestionarios, uno para el "grupo profanos" - Cuestionario tipo A-, y otro para el "grupo instruidos" - Cuestionario tipo B-. Las preguntas de ambos cuestionarios fueron sencillas y quedaron enmarcadas en alguna de las tres dimensiones de la comunicación. Con ello nos fue posible tener parámetros coherentes de valoración del diseño cartográfico y del carácter comunicativo del tema clima de atlas cubano y español (Figura 3).

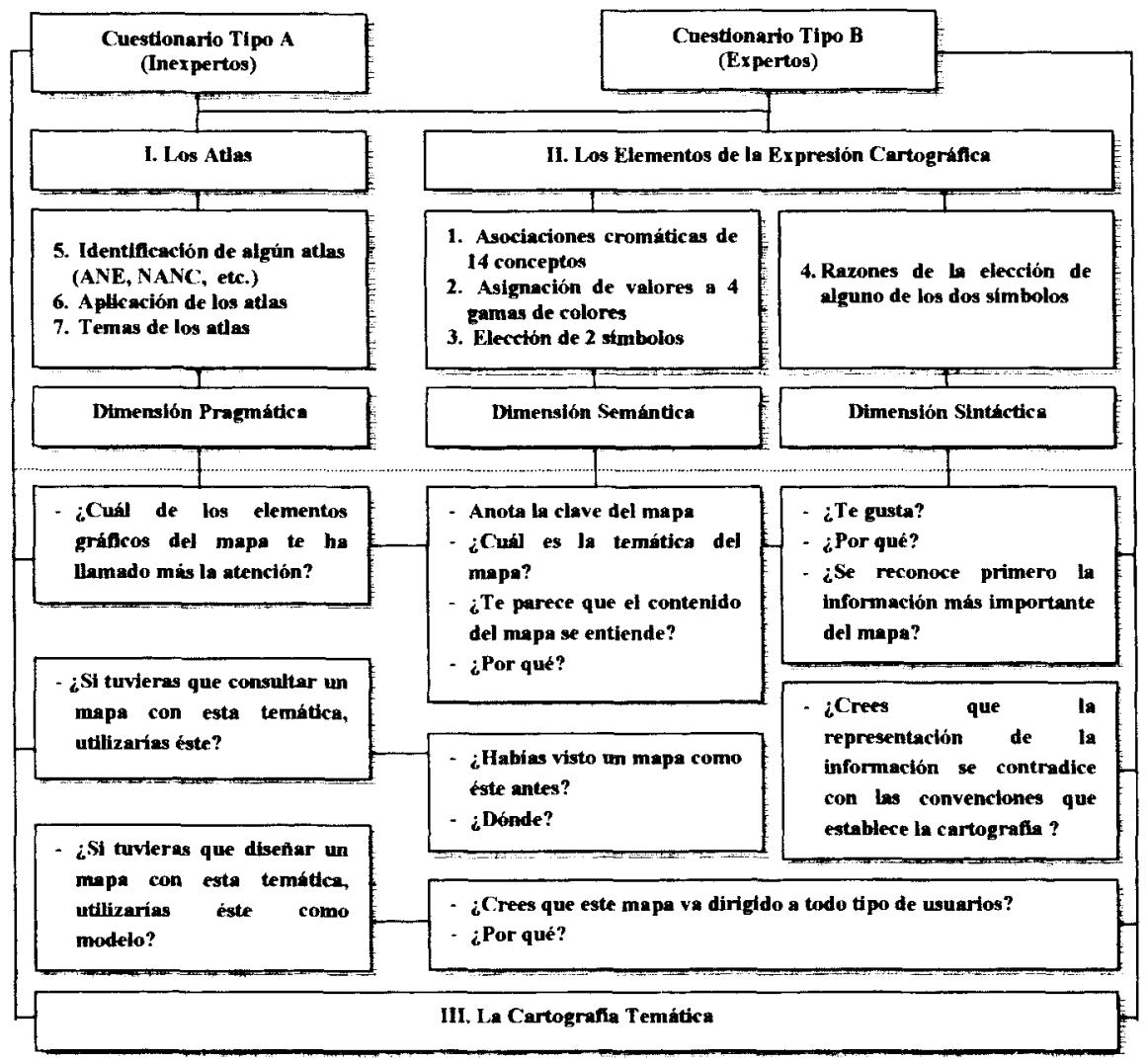

Figura 3. Diseño de los cuestionarios. 


\section{RESULTADOS Y DISCUSIÓN}

A continuación exponemos los resultados más significativos que obtuvimos para los dos atlas, primeramente los del método científico-técnico y después los del método comunicativo-perceptivo ${ }^{3}$.

\section{Método Científico-Técnico}

1. Del contenido temático:

Con respecto el tratamiento científico en sus aspectos temáticos, cabe decir que el Atlas de Cuba es más rico en contenido que el de España, prueba de ello es la serie de mapas de Cartografía Aplicada donde convergen diversas variables, resultado de las investigaciones bioclimáticas que se han realizado en Cuba, y que suponen un valioso apoyo en la planificación de actividades socioeconómicas. Otro ejemplo son los mapas de precipitación cartografiados para dos períodos de referencia (19311972 y 1964-1983), que favorecen la comprensión de este elemento climático en su dimensión temporal.

2. ${ }^{\circ}$ De la estructura sintáctica general:

En la Tabla 2 se resume la estructura temática de ambos documentos; el orden que muestran es similar, comenzando con el mapa de "Clasificación

Tabla 2. Grandes grupos temáticos del clima en los atlas de España y Cuba

\begin{tabular}{cll}
\hline Orden & Atlas Nacional de España & \multicolumn{1}{c}{ Nuevo Atlas Nacional de Cuba } \\
\hline 1. & Clasificación climática & Zonificación, regionatización y clasificación climática \\
$2 .^{\circ}$ & Precipitación & Redes de Observación \\
$3 .^{\circ}$ & Hidrometeoros & Días despejados e insolación \\
$4 .^{\circ}$ & Temperatura & Radiación solar \\
$5 .^{\circ}$ & Humedad relativa & Situaciones sinópticas y trayetoria de los huracanes \\
6. & Evaporación & Presión y vientos \\
$7 .^{\circ}$ & Horas de sol despejado & Temperaturas \\
8. & Radiación solar & Precipitaciones y eventos notables \\
$9 .^{\circ}$ & Presión y vientos & Humedad relativa, turbonadas y humedecimiento \\
10. & Isocronas & Evaporación \\
11. & Situaciones sinópticas & Cartografía aplicada \\
\hline
\end{tabular}

3 Es deseable que para seguir los comentarios que aquí expresamos el lector tenga, al menos, alguno de los documentos, bien sea el Atlas de España o el de Cuba. 
Climática", y sucediéndole a continuación series diferentes por su contenido. Además, los dos atlas tienen el mismo esquema de exposición, primero un texto explicativo, donde se describen las características generales de la variable clima, y a continuación de éste se presenta la serie cartográfica. Sólo en el atlas de España se incluyen algunos textos explicativos que acompañan a los mapas.

\section{De las opciones lingüísticas:}

Según el número de mapas elaborados en cada atlas y las escalas utilizadas, el atlas español (III mapas y 4 escalas distintas) refleja un menor variabilidad $3,6 \%$ con respecto a un $19,7 \%$ del atlas cubano (66 mapas y 13 escalas distintas). Es importante señalar que uno de los problemas de éste último es, precisamente, esta diversidad de escalas que pueden ocasionar problemas al comparar distintos mapas, un ejemplo son los mapas de "Precipitación Media en el Período Seco. 1931-72" y "Precipitación Media en el Período Lluvioso. 1931-72" (pág. V1.3.2) elaborados a escala 1:2.000.000, mientras que los análogos de 1964-33-se han cartografiado a 1:3.000.000 (pág. VI.3.3). Otro problema, es la relación entre categorías, ya que siendo las mismas, no se aplicó la misma gama de color. Esto, además de complicar la comparación de los contenidos, ha supuesto un doble esfuerzo en el trabajo de edición.

En lo referente a las opciones lingüisticas, en la Tabla 3 mostramos la frecuencia de uso de las variables visuales. Además, presentamos tres gráficos complementarios que permiten la comparación porcentual de las opciones coincidentes (Figura 4). Esto define, de alguna manera, el estilo y preferencias de los redactores cartógrafos. Por ejemplo, contavilizando los elementos cartográficos del Atlas español se ve que han preferido la variable visual color, en cambio los autores del Atlas cubano, además del color, utilizan tramas y una mayor variedad de símbolos. En cuanto a los sistemas de implantación, la relación porcentual es casi similar para los de tipo lineal y zonal, donde se marca la diferencia es en el sistema puntual como consecuencia de lo antes mencionado (figuras 4a, 4b).

En los sistemas de representación encontramos pocas diferencias. La principal es el uso exclusivo de cartodiagramas, cartogramas y coropletas en el atlas cubano. En cuanto al resto de sistemas, los de mayor frecuencia son las isolíneas (España) y el dasimétrico (Cuba), conceptualmente estos sistemas son iguales, delimitan superficies estadisticas, aunque la apariencia gráfica sea diferente (Figura 4c). 


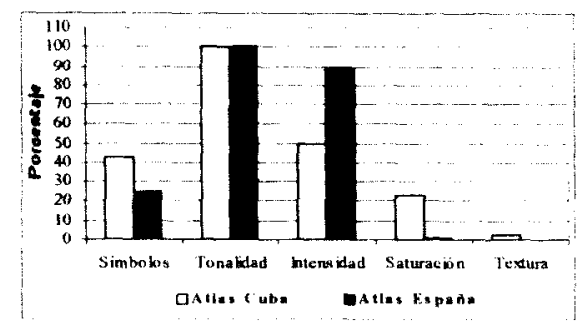

Figura 4a. Elementos cartográficos.

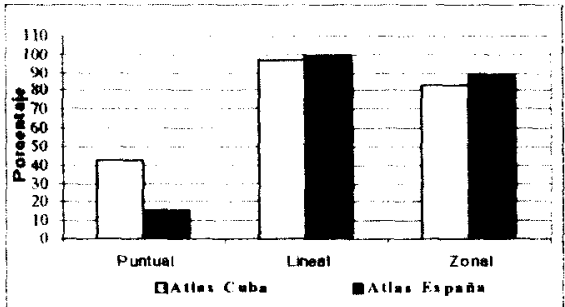

Figura 4b. Sistemas de implantación.

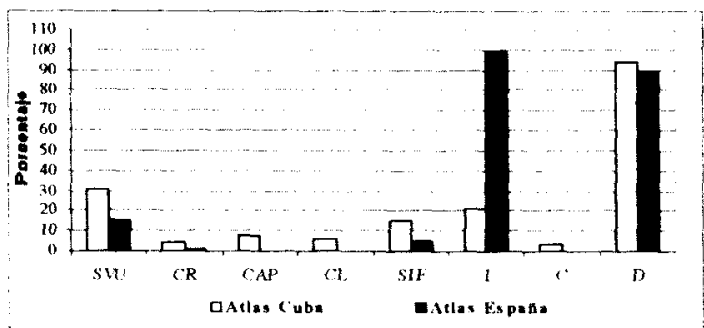

SVU: Simbolos cualitativos de valor unitaries

('R: Cartodiagramas radiales o polares

CAP: Cartogramas de anillas ptoporcionates

Ci. Cartodiagramas lineaies

SLF: Simbolos tineales de flujus

1: Isolineas

C: Corspleticos

1): Dasimetricess

Figura $4 c$. Métodos de representación.

Tabla 3. Opciones lingüisticas empleadas según atlas

\begin{tabular}{|c|c|c|c|c|c|}
\hline \multirow{2}{*}{\multicolumn{2}{|c|}{ Estructura de la Expresión Cartográfica }} & \multicolumn{2}{|c|}{ Atlas España } & \multicolumn{2}{|c|}{ Atlas Cuba } \\
\hline & & \multirow{2}{*}{$\frac{f(x)}{14}$} & \multirow{2}{*}{$\frac{f(\%)}{12,6}$} & \multirow{2}{*}{$\frac{f(x)}{28}$} & \multirow{2}{*}{$\frac{f(\%)}{42,4}$} \\
\hline \multirow{5}{*}{ ELEMENTOS CARTOGRÁFICOS } & Símbolos & & & & \\
\hline & Tonalidad & 111 & 100,0 & 66 & 100,0 \\
\hline & Intensidad & 94 & 84,7 & 33 & 50,0 \\
\hline & Saturación & 4 & 3,6 & 15 & 22,7 \\
\hline & Trama & 0 & 0,0 & 2 & 3,0 \\
\hline \multirow{3}{*}{ SISTEMAS DE IMPLANTACIÓN } & Puntual & 17 & 15,3 & 28 & 42,4 \\
\hline & Lineal & 110 & 99,1 & 64 & 96,9 \\
\hline & Zonal & 99 & 89,2 & 55 & 83,3 \\
\hline \multirow{8}{*}{ MÉTODOS DE REPRESENTACIÓN } & Simbolos de valor unitario & 17 & 15,3 & 20 & 30,3 \\
\hline & $\begin{array}{l}\text { Simbolos de series temporales y } \\
\text { direccionales }\end{array}$ & 1 & 0,9 & 3 & 4,6 \\
\hline & Cartodiagramas & 0 & 0,0 & 4 & 6,1 \\
\hline & Cartogramas & 0 & 0,0 & 5 & 7,6 \\
\hline & Simbolos lineales de flujo & 6 & 5,4 & 10 & 15,2 \\
\hline & Isolineas & 110 & 99,1 & 14 & 21,2 \\
\hline & Dasimétricos & 99 & 89,2 & 62 & 93,9 \\
\hline & Coroplético simple & 0 & 0,0 & 2 & 3,0 \\
\hline
\end{tabular}


4. ${ }^{\circ}$ Del diseño cartográfico y características técnicas:

Respecto al diseño cartográfico, las soluciones son muy diferentes. En general, observamos una adecuada esquematización y aprovechamiento al máximo de los formatos, con una mayor variedad en el Atlas de Cuba que en el de España. Esto, sin duda, ha provocado que al final este segundo documento tenga una presentación más simétrica y agradable.

En cuanto a la legibilidad, cabe señalar que en términos generales los atlas la cumplen, aunque en algunos casos la retención de información y la estimación de los valores no es fácil. Por ejemplo, en algunos mapas del Atlas de España la separación de categorías no es sencilla, pues a veces la intensidad de sus tonos son muy próximas. Un caso concreto es el mapa de "Precipitación Media Anual» que tiene 11 categorías (pág. 9.3), surgiendo la confusión a partir de la séptima. En el resto de mapas encontramos que la discriminación a partir de la quinta o sexta categoría se hace difícil. Al parecer los autores se percataron de este problema y han introducido isolíneas, como guías complementarías para decodificar la información, pues sin ellas se presentarían serios problemas de lectura.

Este problema se da también en 15 mapas del atlas de Cuba. Uno de ellos es el de "Redes de Observación Meteorológica" (pág. VI.L3), en donde las provincias con 17 a 20 y $>20$ pluviómetros por cada 400 $\mathrm{km}^{2}$, no se identifican rápidamente. Además, los símbolos de valor unitario de este mapa, que representan la clase de observación según estación meteorológica, no son fácilmente leíbles, pues el lector tiene que hacer un esfuerzo para descifrar sus valores, ya que se enfrenta a dos tipos de relaciones: la clase de estación y el tipo de observación. En la primera no presentan mayor problema: las formas son excluyentes entre sí; en la segunda, sin embargo, las tres gamas de colores son demasiado próximas y dadas las dimensiones de cada símbolo, no es fácil asociarlas con éstas.

Por último queremos destacar un detalle de orden técnico que hace referencia a las leyendas. En los mapas de España se han empleado las mismas gamas de color por temas relacionados; esto, además de favorecer la comparación, facilitó la edición (selección y separación de color). En cambio, en el Atlas de Cuba se ha elaborado una paleta distinta para cada mapa, aún cuando se tratan el mismo tema; esto, como sabemos, incrementa el coste de la edición e impresión de la obra. 
Valoración final:

Cabe destacar, de estrada, la precisión cartográfica, cualidad presente en la mayoría de los mapas, de manera que el lector puede situar correctamente la variable temática representada. Además es evidente el exhaustiva trabamiento de la información de origen y el empleo adecuado de las bases cartográficas, las que se han generalizado de acuerdo con las características temáticas, aunque observamos notables diferencias en las soluciones dadas. Por ejemplo, en la mayoría de los mapas del atlas cubano se han dibujado los canevás, elemento que en un sentido tradicional es obligado incluir y que en el Atlas de España se omite.

En todos los mapas del Atlas de Cuba se han localizado puntos de referencia, principalmente estaciones meteorológicas, que contribuyen en la interpretación del resto de temas; sin embargo, esto no se estimó necesario para el atlas español, si bien se han trazado los límites administrativos de las Comunidades Autónomas. Además, en los casos de información no del todo precisa existe una nota explicativa en la leyenda (en el caso cubano), o Wen en el texto introductorio (en el caso español). Esta denota la seriedad de los dos documentos y la preocupación por darle al usuario la mayor información de referencia posible para que pueda leer el mapa. Hay que decir, no obstante, que para alcanzar el éxito en la lectura de la obra es de suma importancia el conocimiento del usuario sobre la variable temática.

Con respecto a los problemas de legibilidad de las leyendas del ANE, señalamos que se resolverian cambiando la intensidad de las gamas o empleando, si es posible, leyendas con menor número de rangos. En el Atlas de Cuba los simbolos graduados por segmentos de los mapas "Condiciones Climáticas para la Vida de la Población en el Periodo Seco y del Período lluvioso" (pág. VI.4.3) se han dividido en cinco segmentos que representan los indicadores climáticos; a su vez cada segmento se subdivide en otros tantos que indican los rangos climáticos de las estaciones cartografiadas El problema de estos símbolos es su dimensión, pues es complicado distinguir cada indicador. La solución pasa por aumentar el tamaño del símbolo al menos en un 30\%, consiguiendo así que sean legible.

En suma, nos encontramos ante dos atlas de estructura clásica y guión de exposición similar. La variable representada es una de las tradicionales en Cartografía, de ahi que las soluciones dadas también tengan mucho en común. Sólo destaca el trabajo de síntesis del atlas cubano frente al atlas español en el tema de Cartografía aplicada. 


\section{Método Comunicativo-Perceptivo.}

Las preguntas de la encuesta dirigida a los alumnos fueron hechas con la intensión de fijar nuestra atención en tres aspectos concretos: conocimiento y aplicación de los atlas, elementos de la expresión cartográfica y análisis especifico de una serie cartográfica formada por 23 mapas, 11 de España y 12 de Cuba.

En cuanto a la Dimensión Pragmática, los atlas que los alumnos conocen y utilizan con mayor frecuencia son los de tipo general o escolar (enciclopédicos). Por lo tanto, valdría la pena que los responsables de atlas temáticos consideren en sus proyectos cartográficos la difusión de las obras, pues muy pocos alumnos mencionaron los atlas objeto de estudio. $Y$ cuando afirmaban conocer el ANE, en realidad se trataba de cualquier otro donde apareciera en su titulo la palabra España.

Este hecho nos hace pensar en el importante papel de los profesores, ya que si éstos los utilizaran como recursos de apoyo didáctico, contribuirian favorablemente a su rentabilización. La mayoría de las alumnos han indicado que los atlas son documentos que les auxilian en el desarrollo de algún trabajo, o bien les son útiles cuando desean resolver dudas concretas, principalmente la ubicación de entidades geográficas; indican que existen temas cuyo tratamiento cartográfico desconocian y que, además, han encontrado mapas de difícil interpretación. Por ello, insistimos en el importante papel de los profesores para la difusión de estas obras, de ellos puede surgir la invitación orientadora que propicie su adecuado conocimiento por parte de los alumnos, lo que contribuye a fomentar la cultura cartográfica y el conocimiento de las características geográficas de un determinado territorio.

En relación al apartado «los elementos cartográficos", hemos obtenido resultados para doce asociaciones cromáticas, cuatro gamas de colores y dos simbolos que en concreto hacen referencia a la velocidad y dirección del viento. En este caso, se pretende calibrar la valoración de las Dimensiones Semántica y Sintáctica de ambos atlas.

Como recordarán, la variable visual que más se utiliza en ambos atlas es el color; por ello, fue interesante conocer la apreciación que de ésta tenían los alumnos. Las asociaciones cromáticas de los elementos climáticos cartografiados sobre las que los alumnos opinaron aparecen en la Tabla 4. En este caso, les pedimos que relacionar conceptos comunes que asociamos con las variables cartografiadas.

En la Figura 5 se muestra el Modelo de Cromaticidad con las asociaciones cromáticas que los alumnos señalaron para cada concepto. Si 
Propuesta metodológica para el análisis cartográfico: ejemplo de apreciación...

Tabla 4. Relación de los conceptos corocromáticos con los grupos temáticos

\begin{tabular}{|c|c|}
\hline Conceptos & àrupo Temático \\
\hline a. Día lluvioso & $\begin{array}{l}\text { Precipitación media mensual, estacional, para los periodos: } \\
\text { seco y lluvioso, media anual, máxima diaria absoluta y eventos } \\
\text { notables. }\end{array}$ \\
\hline b. Dia seco & Dias de sol depejado. \\
\hline c. Día frio & Temperatura mínima absoluta, media de la mínima estacional. \\
\hline d. Día templado & Temperatura media mensual, estacional y anual. \\
\hline e. Dia caluroso & Temperatura máxima absoluta, media de la máxima estacional. \\
\hline $\begin{array}{l}\text { f. Dia con nieve } \\
\text { g. Día con granizo } \\
\text { h. Día con tormentas } \\
\text { i. dia con niebla }\end{array}$ & Hidrometeoros \\
\hline j. Día húmedo & Humedad relativa media estacional y anual. \\
\hline k. Evaporación & Evaporación media estacional y anual. \\
\hline 1. Insolación & Insolación media y anual. \\
\hline m. Radiación & Radiación media estacional y anual. \\
\hline n. Presión & Presión media estacional y anual. \\
\hline
\end{tabular}

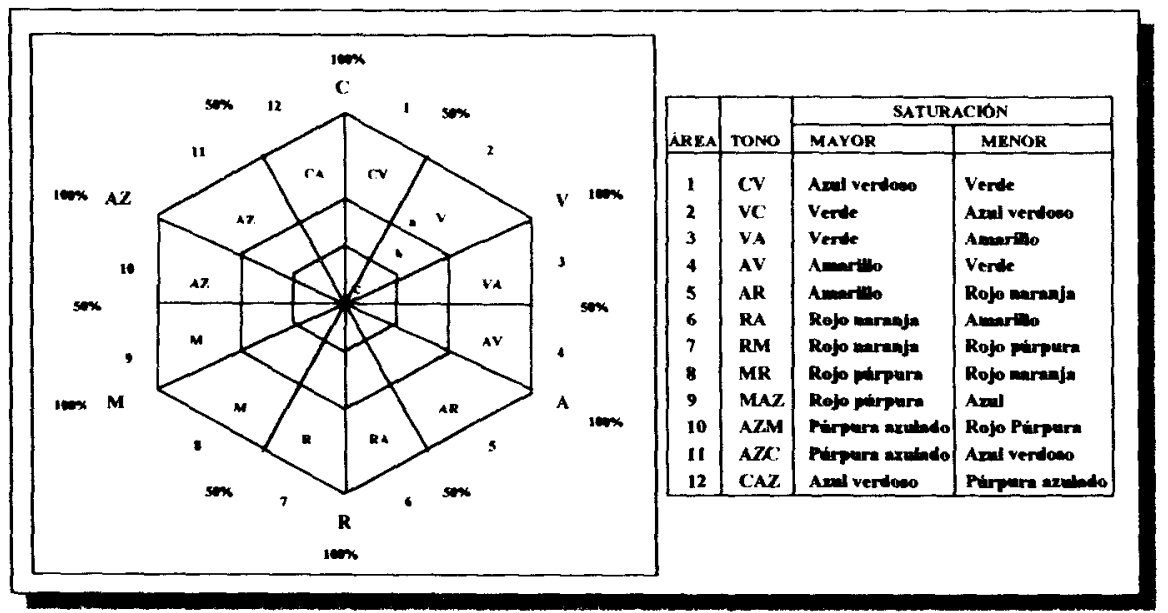

Figura 5. Modelo de cromaticidad (basado en el de Sanz, 1985). 
relacionamos este modelo con la Tabla 4 y la Figura 6, apreciamos que de los 14 conceptos la mayoría coinciden sólo en cinco (día frío, día templado, día caluroso, humedad y presión). Esto nos demuestra la gran diversidad de significación del color. Únicamente en algunos mapas del ANE se aplicó el color que los alumnos han indicado, éstos son: "Número de dios con Temperatura Minima Menor Igual a $0{ }^{\circ} \mathrm{C}$ " (pág. 9.16), en tonos púrpura azulado azul verdoso en baja intensidad (11a); "Temperatura Media de los Meses de Junio, Julio, Agosto y Septiembre" (pág. 9.12-13), en tonos amarillo-rojo naranja en intensidad media (5b); "Temperatura Media de las Máximas. Verano y Otoño", y "Temperatura Máxima Absoluta» (pág. 14), en tono rojo naranja-amarillo en intensidad baja (6a).
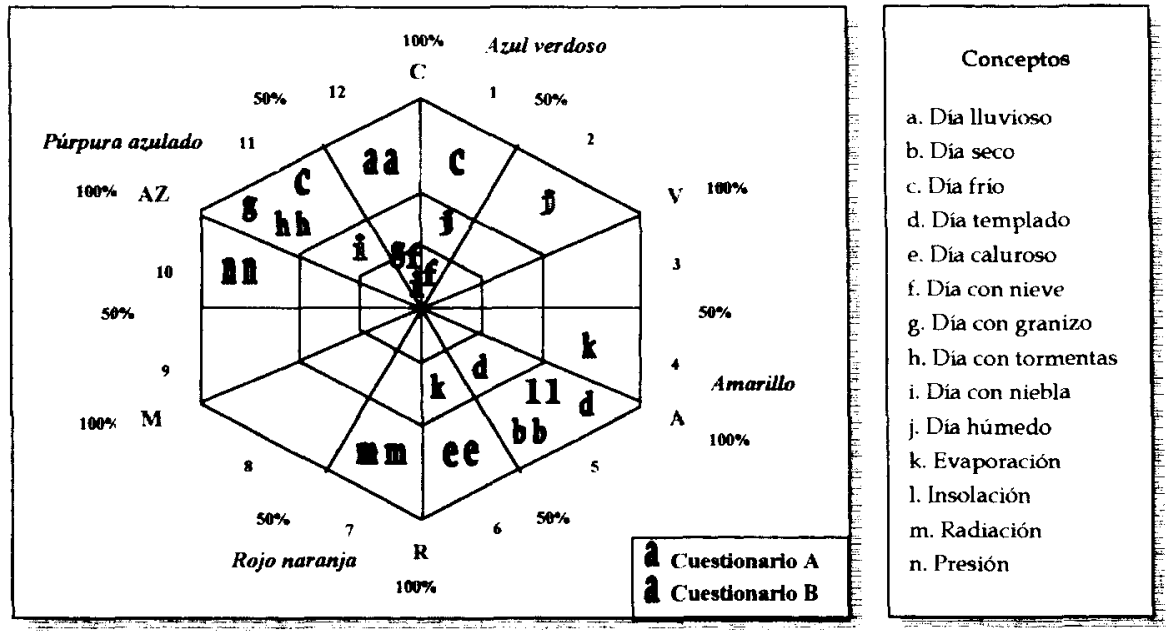

Figura 6. Asociaciones cromáticas que los encuestados indicaron.

Otra particularidad que merece la pena señalar es el resumen de las asociaciones cromáticas que los alumnos esperan ver en estos mapas dado su poder evocador según el tema (véase Figura 6). Se mide, así, la sintonía de su opinión con la de los ejecutores del atlas.

En cuanto a los dos símbolos, el del ANE se distinguió por su diseño, mientras que en el atlas de Cuba se aprecia su legibilidad. Los alumnos han señalado a éste último como el de mejor simbologia, lo que nos indica que para ellos es más importante la relación coherente entre el signifjcante y el significado que su diseño. En la Tabla 5 se aprecia el porcentaje de elección entre uno y otra símbolo. 
Tabla 5. Porcentajes según la elección de los dos simbolos evaluados

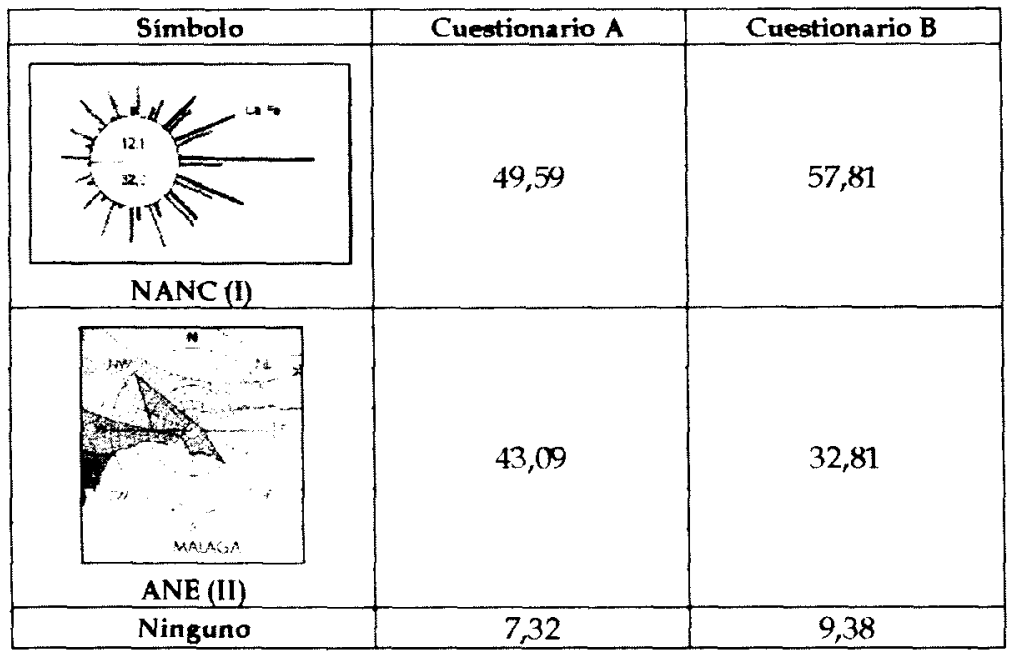

Por último, respecto al apartado "la cartografía temática", no se les pidió a los alumnos su opinión sobre los 117 mapas de la sección clima de los dos atlas. En aras de la rapidez y dado que no se disponía de material suficiente elegimos únicamente aquellos que pudieran compararse, es decir, los de la misma temática, éstos son los que aparecen en Tabla 6.

Una vez realizado el análisis concluimos que las mapas aceptados por cumplir en su totalidad con las tres dimensiones de la comunicación fueron las siguientes:

- Mapas del Nuevo Atlas Nacional de Cuba:

Cuestionario A: Diez responden favorablemente a la Dimensión Semántica y nueve a la Sintáctica y Pragmática.

Cuestionario B: Siete cumplen con la Dimensión Semántica, nueve con la Sintáctica y ocho con la Pragmática.

- Mapas del Atlas Nacional de España:

Cuestionario A: Nueve se han calificado favorablemente en la Dimensión Semántica, siete en la Sintáctica y seis en la Pragmática.

Cuestionario B: Siete mapas que cumplen con la Dimensión Semántica y cinco con la Sintáctica y Pragmática.

También nos interesaba saber qué mapas tuvieron un grado de aceptación mayor para cada grupo de alumnos. Así, en la Figura 7, con un dia- 
Tabla 6. Serie cartográfica evaluada. Mapas comparados

\begin{tabular}{|c|c|c|c|c|}
\hline \multirow{2}{*}{ Grupo temático } & \multicolumn{2}{|r|}{ Mapas del CCANE } & \multicolumn{2}{|r|}{ Mapas de la SCNANC } \\
\hline & No. ${ }^{*}$ & Título & No. ${ }^{*}$ & Título \\
\hline Clasificación climática & $1 \mathrm{EC}$ & Clasificación Climática según Köppen & $1 \mathrm{CC}$ & $\begin{array}{l}\text { Regionalización Climática y } \\
\text { Tipo de Clima }\end{array}$ \\
\hline Precipitaciones & $2 \mathrm{EC}$ & Precipitación Media Anual & $31 \mathrm{CC}$ & Precipitación Media Anual. 1931-72 \\
\hline \multirow{5}{*}{ Temperaturas } & $45 E C$ & Temperatura Media de enero & $23 \mathrm{CC}$ & $\begin{array}{l}\text { Temperatura Media del aire en } \\
\text { enero (invierno) }\end{array}$ \\
\hline & $51 E C$ & Temperatura Media de julio & $24 \mathrm{CC}$ & $\begin{array}{l}\text { Temperatura Media del aire en } \\
\text { julio (verano) }\end{array}$ \\
\hline & $57 \mathrm{CC}$ & Temperatura Media del año & $22 \mathrm{CC}$ & Temperatura Media Anual del aire \\
\hline & $69 \mathrm{EC}$ & Temperatura Máxima Absoluta & $29 C C$ & $\begin{array}{l}\text { Medias de las Temperaturas Míni- } \\
\text { mas Absolutas Anuales del aire }\end{array}$ \\
\hline & $70 \mathrm{EC}$ & Temperatura Mínima Absoluta & $27 \mathrm{CC}$ & $\begin{array}{l}\text { Medias de las Temperaturas } \\
\text { Máximas Absolutas del aire }\end{array}$ \\
\hline \multirow{2}{*}{ Humedad relativa } & \multirow{2}{*}{$75 \mathrm{EC}$} & \multirow{2}{*}{ Humedad Relativa Media Anual } & $48 \mathrm{CC}$ & $\begin{array}{l}\text { Humedad Relativa Media Anual } \\
\text { de las 7:00 horas }\end{array}$ \\
\hline & & & $49 \mathrm{CC}$ & $\begin{array}{l}\text { Humedad Relativa Media Anual } \\
\text { de las 13:00 horas }\end{array}$ \\
\hline Evaporación & $80 \mathrm{EC}$ & Evaporación Media en Anual & $52 \mathrm{CC}$ & Evaporación Media Anual \\
\hline Horas de Sol Despejado & $85 \mathrm{EC}$ & Insolación Anual & $5 \mathrm{CC}$ & Insolación Anual \\
\hline Radiación Solar & $90 \mathrm{EC}$ & Radiación Solar Global Anual & $6 \mathrm{CC}$ & $\begin{array}{l}\text { Radiación Solar Global Media } \\
\text { Anual }\end{array}$ \\
\hline
\end{tabular}

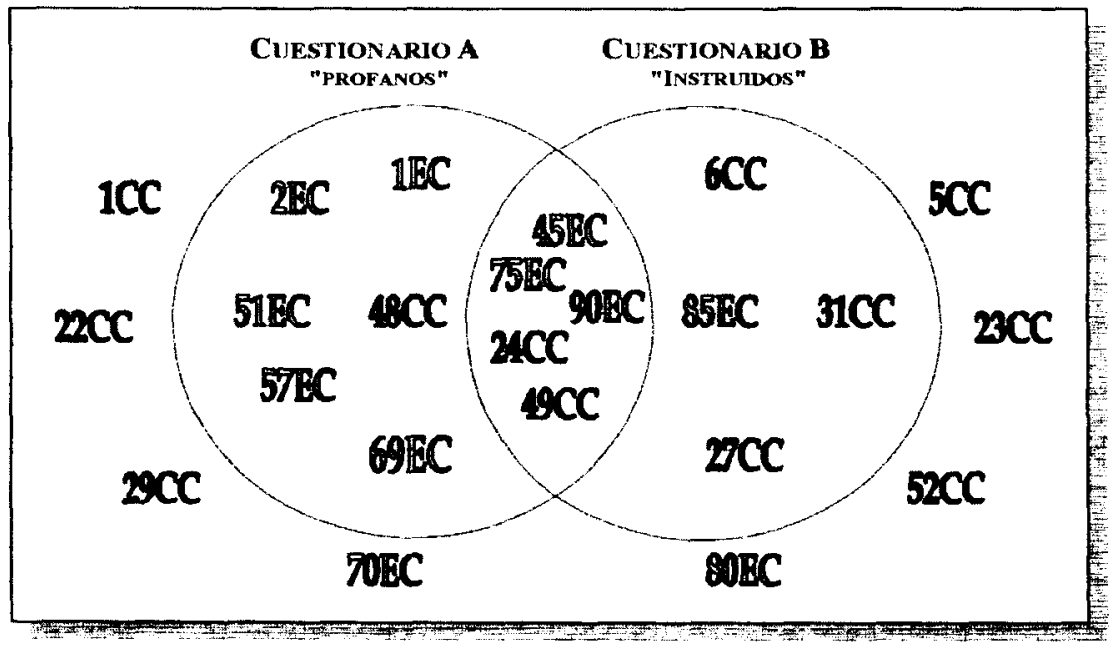

Figura 7. Diagrama de Venn. Intersección por Mapa y Cuestionario $(A, B)$. 
grama de Venn mostramos los mapas que cumplen can las tres dimensiones de la comunicación; de éstos, tres pertenecen al Atlas de España y dos al Altas de Cuba, los mapas son: «Temperatura Media de Enero"(45EC, p. 9.12), "Humedad Relativa Media Anual" (75EC, p. 9.18), «Radiación Solar Global Anual» (90EC, p. 9.21), "Temperatura Media del Aire en Julio (verano)" (24CC, p. VI.2.4), y "Humedad Relativa Media Anual de las 13:00 Horas» (49CC, p. VI.4.1), respectivamente.

Los resultados se resumen en tres categorías (Figura 7):

a) Cinco mapas que cumplen con las exigencias de ambos grupos (intersección).

b) Diez Mapas que cubren las expectativas de alguno de los grupos ( $A$ y B no intersección). Seis para el grupo "profanos" y cuatro para el de «instruidos".

c) Ocho mapas que presentan problemas en algunas de las dimensiones de la comunicación

Con ello, concluimos que, en términos generales, la serie cartográfica evaluado está bien ejecutada, algo más del $65 \%$ de los mapas cumplen su función comunicativa. Sólo ocho presentan problemas en alguna de las dimensiones, se corresponden al Atlas de Cuba y dos al de España. La diferencia numérica se debe, quizá, a que los alumnos encuestados están menos familiarizados con los mapas cubanos.

\section{CONCLUSIONES}

Hasta la fecha no existen estudios sistemáticos de crítica cartográficas que se haya aplicado a los atlas temáticos. La metodología que hemos expuesto pretende ser una aportación para abordar el análisis de estos documentos con dos métodos que se complementan: científico-técnico y comunicativo-perceptivo.

La aplicación de estos métodos nos ha permitido obtener una radiografía de la expresión cartográfica de la variable temática clima del Atlas Nacional de España y del Nuevo Atlas Nacional de Cuba, lo que demuestra su validez y, sobre todo, su adaptabilidad a estudios particulares y más en concreto a su utilización didáctica.

Destacamos que ambos atlas abordan con cierta amplitud la descripción y representación de las principales variables climáticas, seleccionadas oportunamente, deseables, referidas a los períodos más representativos (mes, estación, año) de cada país, elaboradas con todos los datos 
disponibles, de calidad suficiente y homologables con los criterios de la Organización Meteorológica Mundial (separación de isolíneas, variables elegidos, etc.).

Los comentarios que hemos expresado en este trabajo, consideran tanto el carácter eminentemente divulgativo de los dos atlas, como la cantidad de información geográfica y el nivel de detalle alcanzado en su manejo. Es importante señalar que toda obra cartográfica contiene una carga subjetiva derivada de la experiencia del grupo de científicos y técnicos que participaron en su confección y su éxito depende, en buena medida, de la formación y atención (apreciación) del lector(a). La subjetividad está determinada por la percepción individual y el conocimiento en estos temas, lo que aconseja no imponer reglas tajantes, más o menos inflexibles, en la elaboración de estas obras cartográficas.

\section{BIBLIOGRAFIA}

Bertin, J. (1973): Sémiologie Graphique. 2. edición. París, Francia. Mouffon-Gauffer-Villars. Bertin, J. (1988): La Gráfica y el Tratamiento Gráfico de la Información. Madrid, España. Taurus.

Brunet, R. (1987): La Carta, Mode d'emploi. París, Francia, Fayard/Reclus.

CUENIN, R (1972): Cartographie Génèrale. Notions Génèrales et Principes d'Elaborations. Collection Scientifique de l'Institute Geographique National. Tomo I. París, Francia. Eyrolles.

Floristan, S. A.; SANCHO, C.-J. (1993): "Gran Atlas de Navarra". Serie Geográfica N. 3. Atlas Regionales. Madrid, España. Universidad de Alcalá, Departamento de Geografía, págs. 35-48.

JOLy, E. (1979): La Cartografía, 1. a edición (versión completa). Barcelona, España. Ariel.

Joly, F. (1988): La Cartografía, 1. ${ }^{a}$ edición en lengua castellana (versión resumida). Barcelona, España. Oikos-Tau.

KraAk, M. J.; Ormeling, F. J. (1996): Cartography. Visualization of spatial data. Harlow, London. Longman.

Monkhouse, F. J.; WILKInson, H. R (1976): Mapas y Diagramas. Barcelona, España. OikosTau.

NÚNEEZ DE LAS, C. R (1993): "Atlas Regional: Lenguaje Cartográfico y Nuevas Orientaciones". Serie Geográfica N. ${ }^{\circ}$ 3. Atlas Regionales. Madrid, España. Universidad de Alcalá, Departamento de Geografia, págs. 9-13.

RAISZ, E. (1985): Cartografía General. 7. ${ }^{a}$ edición. Barcelona, España. Omega.

RoBinson, H. A. (1987): Elementos de Cartogratía. Barcelona, España. Omega.

RoBINSON, H. A. (1995): Elements of Cartography. US.A., John Wiley \& Sons, Inc.

SAlitCHEV, K. A. (1981): Cartografía. La Habana, Cuba. Pueblo y Educación.

SANCHO, C. J. (1993): “Atlas Temáticos Regionales: Opciones Sintácticas". Serie Geográfica N. ${ }^{\circ}$ 3. Atlas Regionales. Madrid. España. Universidad de Alcalá, Departamento de Geografia, págs. 15-34.

SANZ, J. C. (1985): El Lenguaje del Color. 1." edición. España. Hermann Blume.

SANZ, N. A. (1993): "Los Contrastes Regionales de España a través del Atlas Nacional de España". Serie Geográfica N. ${ }^{\circ}$ 3. Atlas Regionales. Madrid, España. Universidad de Alcalá, Departamento de Geografia, págs. 57-65.

Vázouez, M. F.; Martin, L. J. (1987): Lectura de Mapas. Ministerio de Obras Públicas y Urbanismo. Instituto Geográfico Nacional. Madrid, España.

Wood, H. C.; Keller, C. P. (1996): Cartographic Design. Theoretical and Practical Perspectives. International Western Geographical Series. Department of Geography, University of Victoria, Victoria, British Columbia. Canadá. John Wiley \& Sons. 


\section{REFERENCIAS CARTOGRÁFICAS}

Atlas Agroclimático Nacional de España, Escala 1:500.000. Ministerio de Agricultura. 1. ${ }^{a}$ edición. Madrid, España, 1979.

Atlas de la Comunidad de Madrid. Consejeria de Política Territorial. Comunidad de Madrid. Fundación Caja Madrid, 1992.

Atlas Nacional de España, Sección II, Grupo 9 CLIMATOLOGIA. Ministerio de Obras Públicas, Transportes y Medio Ambiente, Dirección General del Instituto Geográfico Nacional (IGN). Madrid, España, 1992.

Atlas Nacional de España, Tomo I, grupos 1-13. Ministerio de Obras Públicas, Transportes y Medio Ambiente, Dirección General del Instituto Geográfico Nacional (IGN). Madrid, España, 1995.

Atlas Nacional de España, Tomo II, grupos 39-45. Ministerio de Obras Públicas, Transportes y Medio Ambiente, Dirección General del Instituto Geográfico Nacional (IGN). Madrid, España, 1996.

Atlas Nacional de España, Tomo IV, grupos 39-45. Ministerio de Obras Públicas, Transportes y Medio Ambiente, Dirección General del Instituto Geográfico Nacional (IGN). Madrid, España, 1997.

Atlas Nacional de México. Instituto de Geografía, Universidad Nacional de Autónoma de México, 1990.

Atlas of Israel. The Survey of Israel, MacMillan Publishing Campany, 1985.

Gran Enciclopedia Larouese. Atlas. 1. ${ }^{a}$ edición, Barcelona, España. Planeta, 1992.

Nuevo Atlas Nacional de Cuba. Instituto de Geografia de la Academia de Ciencias de Cuba, Instituto de Geodesia y Cartografía. La Habana, Cuba, 1989.

The National Atlas of the United States of America. Department of Interior of the United States of America. Geological Survey, Washington D.C., 1970. 